\title{
Space-division multiplexed transmission over few-mode- and coupled-core fiber based on coherent MIMO digital signal processing
}

Roland Ryf, Sebastian Randel, René-Jean Essiambre, Peter Winzer

Roland Ryf, Sebastian Randel, René-Jean Essiambre, Peter J. Winzer, "Space-division multiplexed transmission over few-mode- and coupled-core fiber based on coherent MIMO digital signal processing," Proc. SPIE 8284, Next-Generation Optical Communication: Components, Sub-Systems, and Systems, 828402 (24 January 2012); doi: 10.1117/12.915671 


\title{
Space-Division Multiplexed Transmission over Few-Mode- and Coupled-Core Fiber Based on Coherent MIMO Digital Signal Processing
}

\author{
Roland Ryf, Sebastian Randel, René-Jean Essiambre, and Peter J. Winzer \\ Bell Laboratories, Alcatel-Lucent, 791 Holmdel-Keyport Rd, Holmdel, NJ, 07733, USA.
}

\begin{abstract}
The capacity of optical transmission systems has increased dramatically since their first deployments in the mid 1970s . However, studies show that the theoretical capacity limit of single-mode fiber is about to be reached, and space-division multiplexing has been proposed to overcome this limit. With the high levels of integration needed for economic deployment, space-division multiplexing may exhibit large crosstalk between the supported fiber modes. We propose to use coherent multiple-input multiple-output (MIMO) digital signal processing (DSP), a technique widely used in wireless communication, to compensate crosstalk present in spatial multiplexing over fibers. According to MIMO theory, crosstalk in multi-mode transmission systems can be completely reversed if the crosstalk is described by a unitary transformation. For optical fibers this is fulfilled if all available fiber modes can be selectively excited and if all the modes are coherently detected at the end of the fiber, provided that mode-dependent loss is negligible. We successfully applied the technique to demonstrate the transmission of six independent mode-multiplexed 20-Gbaud QPSK signals over a single, optically amplified span of 137$\mathrm{km}$ few-mode fiber (FMF). Further, in a multi-span experiment, we reach $1200 \mathrm{~km}$ by transmitting over a 3-core coupled-core fiber (CCF). Details for both experiments will be presented, including the description of the supported polarization- and spatial modes of the fiber, the mode multiplexers used to launch and detect the modes, and the MIMO DSP algorithm used to recover the channels.
\end{abstract}

Keywords: Mode-multiplexing, MIMO, coherent communication, multi-mode fiber, multi-core fiber

\section{INTRODUCTION}

Single-mode fibers (SMFs) have been successfully exploited for long-haul optical transmission for over two decades, during which time their capacity continuously grew by three orders of magnitudes. The growth was fueled by the successive introduction of wavelength-division multiplexing (WDM), polarization-division multiplexing (PDM), and higher-order modulation formats. ${ }^{1}$ However the capacity of SMFs is now approaching the limits imposed by the combination of Shannon's information theory and nonlinear fiber effects. ${ }^{2}$ In order to continue to grow the capacity and fulfill demands, a new dimension is now required and it has been suggested ${ }^{3}$ that space-division multiplexing (SDM) be explored as a technique to extend the growth in capacity in optical transmission systems. In SDM, spatially diverse paths are used to transmit multiple channels, and if realized over a single fiber, SDM offers a significant potential for cost-, space- and energy savings. ${ }^{4}$

SDM over a single fiber can be achieved in two ways. The first approach consists of using waveguides that support multiple waveguide modes, such as in a multimode fiber (MMF). Earlier attempts of SDM over $\mathrm{MMF}^{5-8}$ were limited in transmission distance and bandwidth, because the waveguide modes could not be selectively excited and detected and also because of the large modal differential group delay (DGD) present in standard MMF. More recently transmission distance and bandwidth has been increased by using few-mode fibers (FMFs) ${ }^{9-14}$ which are MMF that support only a small number of waveguide modes. In this work the FMF transmission distance is further extended up to $137 \mathrm{~km}$ at $240 \mathrm{Gbit} / \mathrm{s}$ single wavelength channel bandwidth. ${ }^{15}$

Further author information: (Send correspondence to R. Ryf)

R. Ryf: E-mail: roland.ryf@alcatel-lucent.com, Telephone: 17328887038

S. Randel: E-mail: sebastian.randel@alcatel-lucent.com, Telephone: 17328887192

R.-J. Essiambre: E-mail: rene.essiambre@alcatel-lucent.com, Telephone: 17328887122

P.J. Winzer: E-mail: peter.winzer@alcatel-lucent.com, Telephone: 17328887210

Next-Generation Optical Communication: Components, Sub-Systems, and Systems, edited by Guifang Li, Dieter Stefan Jäger, Proc. of SPIE Vol. 8284, 828402 · C 2012 SPIE CCC code: $0277-786 X / 12 / \$ 18 \cdot$ doi: $10.1117 / 12.915671$ 
For such a distance significant crosstalk between all modes supported by the FMF can be observed, and MIMO DSP for crosstalk mitigation is required.

The second approach to implement SDM consists of multiple spatially separate parallel waveguides formed inside the fiber. The simplest implementation is represented by the multi-core fiber (MCF), and consists of multiple cores distributed across the fiber section. In this approach it is desirable to minimize the crosstalk between cores so that the individual cores can be treated as individual channels, thus greatly simplifying the communication system design. Despite having the cores spatially separated, achieving low crosstalk between cores for long distance transmission, can be a challenging task because the light is not completely confined in the cores. However recently low crosstalk 7 -core MCFs have been demonstrated ${ }^{16,17}$ using core spacings of $>45 \mu \mathrm{m}$ and non-standard cladding diameters $>150 \mu \mathrm{m}$. In this work we show that the fiber design can be drastically simplified, and the spacing between cores reduced to $<30 \mu \mathrm{m}$ if crosstalk is allowed and subsequently undone by multiple-input multiple-output (MIMO) digital signal processing (DSP). Despite the very large crosstalk between cores present in the CCF, we have shown that a transmission distance of over $1200 \mathrm{~km}$ can be achieved. ${ }^{18}$

The general method to perform SDM transmission over FMF or CCF in the presence of crosstalk or coupling between the SDM channels is shown in Fig. 1. The signals from $N$ transmitters are coupled into the SDM

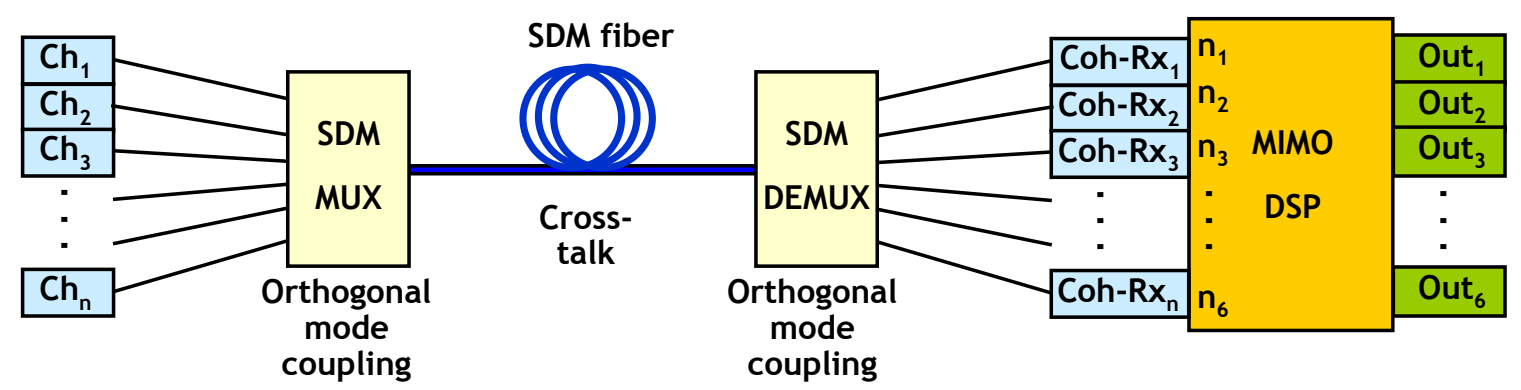

Figure 1. $N \times N$ coherent SDM transmission based on MIMO DSP.

fiber by an SDM multiplexer (SDM-MUX). After transmission through the SDM fiber, the received signals are demultiplexed by a SDM demultiplexer (SDM-DEMUX) and fed into $N$ coherent receivers. The received signals are subsequently processed using MIMO DSP. In order to achieve the full SDM capacity gain of a factor $N$ at high reliability (i.e. low outage), it is required that the $N \times N$ transmission channel consisting of SDM-MUX, SDM fiber, and SDM-DEMUX be described by a unitary linear transfer function. ${ }^{19}$ In particular this requires that the SDM-MUX and DEMUX be capable of exciting all the modes supported by the SDM-fiber in a selective way ${ }^{*}$. For the SDM fiber, the requirement implies negligible polarization dependent loss (PDL) and negligible mode-dependent loss (MDL). These conditions are fulfilled for both the FMF and CCF presented in this work.

The paper is organized as follows: In Sec. 2 we describe SDM experiments performed in FMF that supports 6 polarization- and spatial-modes. Sec. 3 describes the extension of the FMF experiments to longer distance by using FMF Raman amplification, whereas Sec. 4 describe transmission experiments performed in 3-core CCFs.

\section{SPACE-DIVISION MULTIPLEXING OVER FEW-MODE FIBER}

The FMF used in this work is based on a depressed cladding index profile with normalized frequency $V \approx 5$, where the normalized frequency is defined as $V=\pi d / \lambda \sqrt{n_{1}^{2}-n_{2}^{2}}$, where $d$ is the core diameter, $\lambda$ the wavelength of light, and $n_{1}$ and $n_{2}$ are the refractive indices of core and cladding, respectively. The fiber was designed to guide exactly six polarization- and spatial-modes (The fundamental $\mathrm{LP}_{01}$ mode and the twofold degenerate $\mathrm{LP}_{11}$ mode), and also to minimize the modal DGD between the $\mathrm{LP}_{01}$ and $\mathrm{LP}_{11}$ across the C-band. The DGD has to be kept as small as possible, because any delay introduced between the SDM channels has to be compensated by means of filters with a correspondingly large memory as part of the MIMO DSP. The fiber employed in our experiments $^{12}$ has a loss coefficient of $0.205 \mathrm{~dB} / \mathrm{km}$ at $1550 \mathrm{~nm}$ and no significant mode-dependent loss. The

\footnotetext{
${ }^{*}$ Here we extend the use of the word "mode" also for the case of CCFs, where it indicates the super-modes ${ }^{20}$ formed by the coupled spatial channels.
} 
effective areas of the $\mathrm{LP}_{01}$ and $\mathrm{LP}_{11}$ modes are approximately 155 and $159 \mu \mathrm{m}^{2}$, respectively, and the chromatic dispersion is $18 \mathrm{ps} /(\mathrm{nm} \mathrm{km})$ for both $\mathrm{LP}_{01}$ and $\mathrm{LP}_{11}$ modes. The DGD between the $\mathrm{LP}_{01}$ mode and the $\mathrm{LP}_{11}$ mode was measured by launching a 100-ps intensity-modulated probe pulse simultaneously into the $\mathrm{LP}_{01}$ and the $\mathrm{LP}_{11}$ spatial mode, and its value measured for a 96-km long FMF was found to be within $2.6 \pm 0.1 \mathrm{~ns}$ over the wavelength range of 1530 to $1565 \mathrm{~nm}$, corresponding to a length specific DGD of $27 \mathrm{ps} / \mathrm{km}$. In comparison, the DGD of a step-index (SI) profile FMF with the same normalized frequency is $4300 \mathrm{ps} / \mathrm{km}$, and therefore more than two orders of magnitude larger. Fiber with DGD even lower than $27 \mathrm{ps} / \mathrm{km}$ are highly desirable in order to further reduce the memory size required in the MIMO DSP.

In order to achieve the maximal SDM capacity gain, the complete set of modes supported by the 6-mode FMF has to be launched without significant crosstalk. ${ }^{19}$ We therefore built spatial-mode multiplexers (MMUXs) to couple the light from three single-mode fibers into the different spatial modes of the 6-mode FMF. A simple way to selectively couple light into a $\mathrm{LP}_{11}$ mode was shown in Refs. 21,22 , where a phase plate having a phase profile matching the phase of the target mode is inserted into the optical path between the incoming SMF and the 6-mode FMF. The theoretical phase and intensity profiles of the linearly polarized (LP) spatial modes of a 6-mode FMF are shown in Fig. 2 d) and c). Coupling into the $\mathrm{LP}_{11}$ mode requires a phase plate that introduces

a)

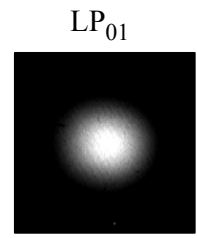

b)

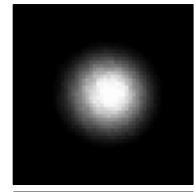

c)

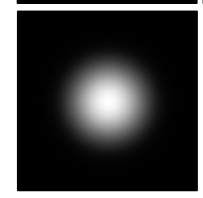

d)

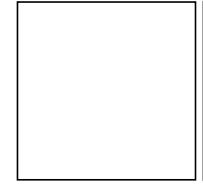

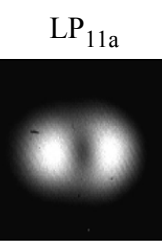
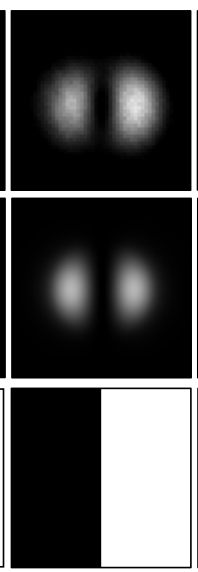
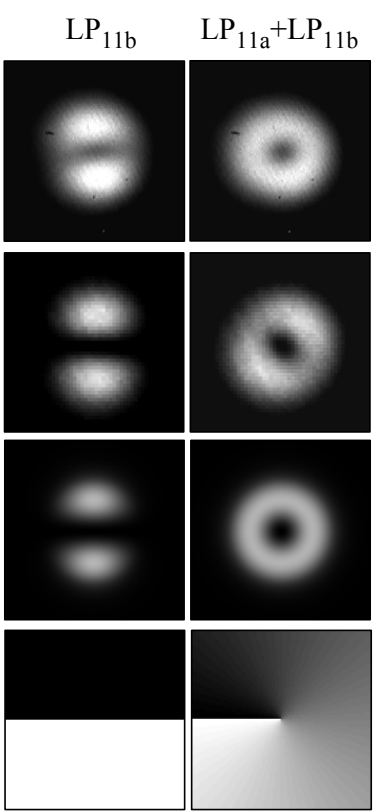

e)

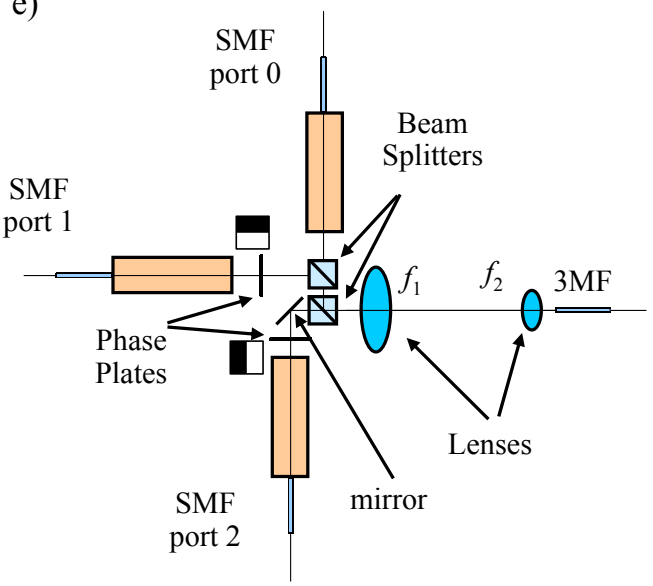

Figure 2. Mode intensity profiles measured after a) 96-km and b) 33-km 6-mode FMF for different launched modes. c) Theoretical mode intensity profiles for a 6-mode FMF and d) corresponding phase profiles. e) Schematic set-up of the 6-mode FMF mode multiplexer (MMUX).

a $\pi$ phase jump between two half planes. Two orthogonal orientations for the half planes are possible, and are denoted as $\mathrm{LP}_{11 \mathrm{a}}$ and $\mathrm{LP}_{11 \mathrm{~b}}$ in Fig. 2, respectively. The $\mathrm{LP}_{01}$ mode, does not require a phase plate and can be directly coupled into the FMF. The coupling efficiency $\eta_{n m}$ describing the the coupling between a SMF and a corresponding $\mathrm{LP}_{n m}$ mode of the FMF can be calculated using the overlap integral ${ }^{23}$

$$
\eta_{n m}=\left|\int_{-\infty}^{+\infty} \int_{\operatorname{SMF}}(x, y) \phi_{n m}(x, y) \psi_{n m}^{*}(x, y) d x d y\right|^{2},
$$

where $\chi_{\mathrm{SMF}}$ is the normalized complex amplitude produced by the SMF on the facet of the FMF, where the normalization $\int_{-\infty}^{+\infty}\left|\chi_{\mathrm{SMF}}(x, y)\right|^{2} d x d y=1$ is used. The transfer function $\phi_{n m}$ describing the phase plate has an amplitude $\left|\phi_{n m}(x, y)\right|=1$ and is set to $\phi_{n m}(x, y)=\psi_{n m}(x, y) /\left|\psi_{n m}(x, y)\right|$, where $\psi_{n m}$ is the normalized complex 


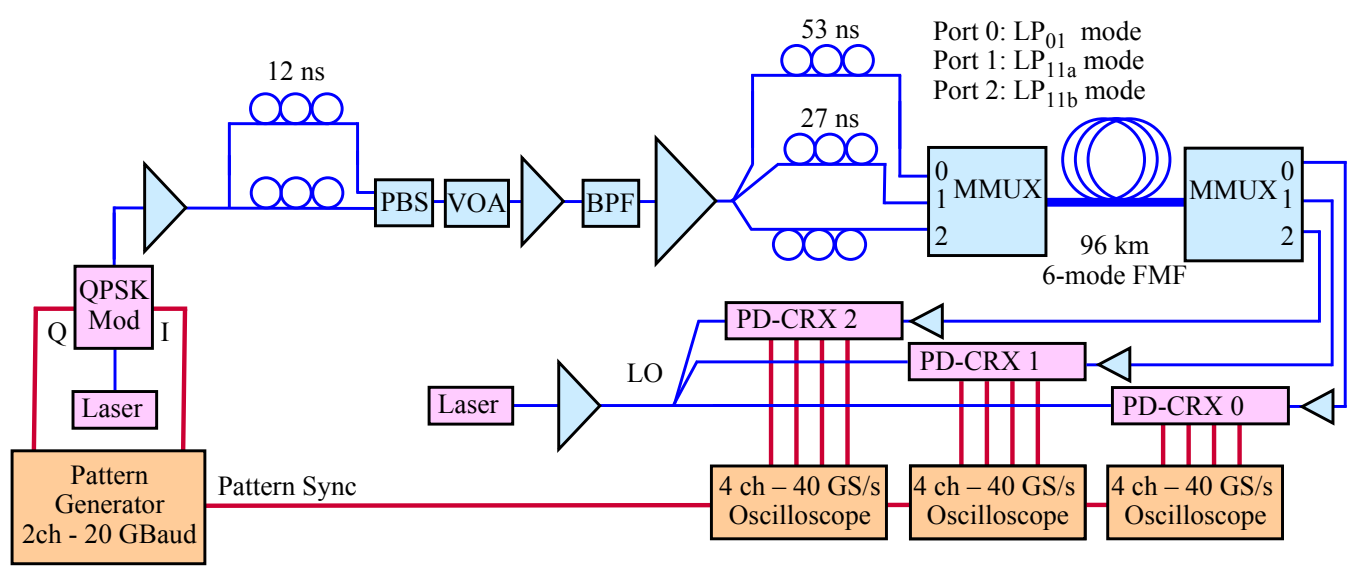

Figure 3. Experimental set-up. VOA: Variable optical attenuator, PBS: Polarization beam splitter,QPSK-Mod: QPSK Modulator, BPF: Bandpass filter, LO: Local oscillator, PD-CRX: Polarization-diversity coherent receiver.

amplitude of the corresponding $\mathrm{LP}_{n m}$ mode, and ${ }^{*}$ indicates complex conjugation. The optimum coupling efficiency $\tilde{\eta}_{n m}$ is found to be nearly $0 \mathrm{~dB}$ for the $\mathrm{LP}_{01}$ mode whereas it is close to $1 \mathrm{~dB}$ for the $\mathrm{LP}_{11}$ mode.

Multiple modes can be excited simultaneously by using beam splitters as shown in Fig. 2 e). The MMUX in Fig. 2 e) has three ports consisting of 3 collimators with a full width half max (FWHM) beam diameter of $500 \mu \mathrm{m}$. The light from port 0 is directly coupled into the $\mathrm{LP}_{01}$ mode of the 6 -mode FMF, and port 1 and 2 have orthogonal phase plates inserted in their optical path and will excite the $\mathrm{LP}_{11 \mathrm{a}}$ and $\mathrm{LP}_{11 \mathrm{~b}}$ spatial modes, respectively. A double telecentric imaging system formed by a lens pair with focal lengths $f_{1}=75 \mathrm{~mm}$ and $f_{2}=3.9 \mathrm{~mm}$, respectively, is used to image the phase plates on the facet of the 6 -mode FMF. The phase plates are fabricated from a Borosilicate glass substrate with a thickness of $0.7-\mathrm{mm}$, by a photolithographic process followed by an etching process. The resulting thickness difference $d$ necessary to produce a phase difference of $\pi$ is given by

$$
d=\frac{\lambda}{2\left(n_{\mathrm{gl}}-1\right)}=1.703 \mu \mathrm{m}
$$

where $n_{\mathrm{gl}}=1.455$ is the refractive index of the Borosilicate glass.

The beams from the three collimators are then combined using two beam splitters with a reflectivity of $50 \%$ and a transmittance of $38 \%$. For this arrangements coupling losses of $9.6 \mathrm{~dB}, 9 \mathrm{~dB}$, and $7.8 \mathrm{~dB}$ are measured for the $\mathrm{LP}_{01}, \mathrm{LP}_{11 \mathrm{a}}$, and $\mathrm{LP}_{11 \mathrm{~b}}$ spatial modes, respectively. Note that with an optimized splitting ratio of the splitters and ideal optical components the predicted minimum loss for the coupler is $5.5 \mathrm{~dB}$.

The modal crosstalk of the MMUX was measured using a pair of MMUXs connected by a short (2-m) 6-mode FMF such that mode coupling inside the fiber can be neglected. A crosstalk suppression from the $\mathrm{LP}_{01}$ spatial mode to the $\mathrm{LP}_{11}$ spatial mode of $>28 \mathrm{~dB}$ was measured.

Also the mode intensity profiles were measured at the end facet of the 6-mode FMF using an InGaAs infrared camera after 33 and $96 \mathrm{~km}$ of fiber. The results are reported in Fig. 2 a) and b), respectively. The measurements after $33 \mathrm{~km}$ FMF are in good agreement with the corresponding simulated intensity profile reported in Fig. 2 b), whereas after $96 \mathrm{~km}$ the images become "blurry" because of crosstalk between the modes. This was also confirmed by measuring the crosstalk of the MMUX pair interconnected by 33 and $96 \mathrm{~km}$ of FMF, where a crosstalk fluctuating around $-18 \mathrm{~dB}$ and $-11 \mathrm{~dB}$, respectively, was observed. Even if the noticeable crosstalk in Fig. 2 a) is small, the crosstalk produces a significant transmission penalty if left uncorrected.

\subsection{Experimental Set-Up for SDM transmission}

The SDM transmission-measurement setup is shown in Fig. 3. The source signal for the experiment is generated by modulating an external cavity laser (ECL) at 1560-nm wavelength and having a linewidth of $100 \mathrm{kHz}$. The signal is modulated by a double-nested $\mathrm{LiNbO}_{3}$ Mach-Zehnder modulator using quadrature-phase-shift-keying (QPSK) where the in-phase (I) and quadrature (Q) components are driven by two independent De Bruijn bit 
sequences (DBBS) of length $2^{12}$, respectively. The use of two independent bit patterns offers the advantage of avoiding correlation effects as described in Refs. 24,25. Subsequently, a polarization-multiplexing stage with a delay of 12 ns generates a PDM-QPSK signal which is followed by a noise-loading section consisting of a variable optical attenuator (VOA) in front of an Erbium-doped fiber amplifier (EDFA). The PDM-QPSK signal is then split into three copies with a relative delay of $27 \mathrm{~ns}$ and $53 \mathrm{~ns}$, that are connected to different SMF ports of the input MMUX. The MMUX is connected to the FMF fiber under test and a second MMUX acting as mode demultiplexer is used to terminate the FMF. The mode demultiplxed signals are then amplified using lownoise EDFAs before being detected by three polarization-diversity coherent receivers (PD-CRX). Each PD-CRX consists of a polarizing beam splitter (PBS) followed by two optical hybrids whose output ports are terminated by four balanced receivers. A second ECL is used as a local oscillator (LO) and the resulting 12 electrical high-speed signals from the PD-CRXs are captured using 3 high-speed digital oscilloscopes with 4 ports each, operating at a sampling rate of $40 \mathrm{GS} / \mathrm{s}$. Each measurement consists of a total of four million samples captured using a common trigger signal.

\subsection{BER evaluation using MIMO DSP}

The measurments are subsequently analyzed using off-line DSP. The $6 \times 6$ MIMO DSP architecture is an extension of the $2 \times 2$ implementation frequently found in coherent PDM receivers and is shown in Fig. 4 a) and b). The six complex received signals $r_{1-6}$ derived from the 12 electrical signals from the three PD-CRXs are

a)

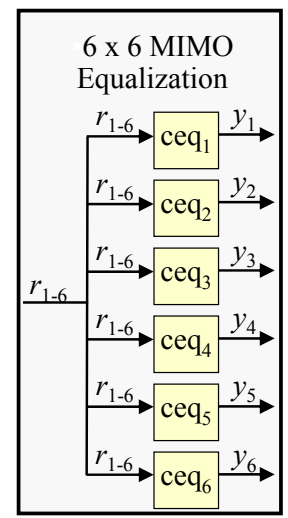

b)

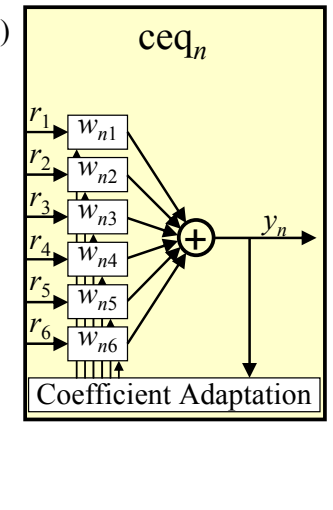

c)

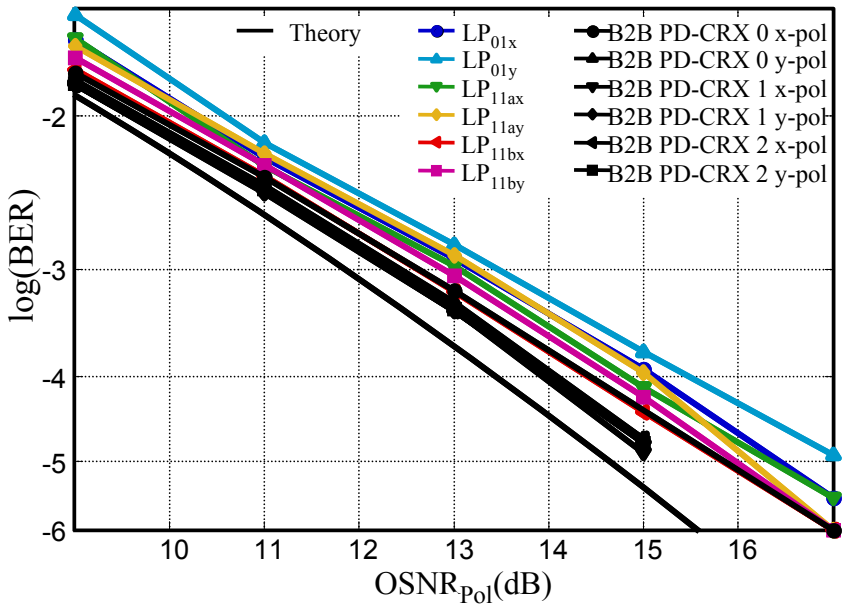

Figure 4. a) Architecture of the MIMO equalization block. b) Architecture a column equalizer $c e q_{n}$. c) BER curves for 6-channel PDM-SDM transmission of 20-Gbaud QPSK over $96 \mathrm{~km}$ of 6-mode FMF. Also shown for reference are the back-to-back measurements and the theoretical limit.

fed into six column equalizers $\left(c e q_{1-6}\right)$ (see Fig. 4 a)). Each of the column equalizers produces a single output signal $y_{1-6}$. The architecture of the column equalizer $\mathrm{ceq}_{n}$ is reported in Fig. 4 b) and contains six feed-forward equalizers (FFEs). Each FFE has $L$ taps associated with the complex coefficient vectors $w_{n 1-n 6}$ with length $L$. The recovered signal $y_{n}$ is determined as the sum of the outputs of the 6 FFEs. The $6 \times 6$ MIMO DSP requires a total of 36 FFEs and the equalizer coefficients $w_{n 1-n 6}$ are optimized by applying the least-mean-square estimate (LMS) algorithm ${ }^{26}$ modified to include carrier phase estimation (CPE) based on the fourth power algorithm. ${ }^{27} \mathrm{In}$ order to achieve initial convergence of the equalizer coefficients, the algorithm assumes knowledge of the received data (data aided) for the first 500,000 symbols, and later switches to a decision-directed LMS algorithm. A detailed description of the algorithm can be found in Ref. 25. Finally the bit-error rate (BER) is evaluated over the last one million bits of the acquired data.

Fig. $4 \mathrm{c}$ ) shows the experimental BER curves after off-line $6 \times 6$ MIMO processing with 120 taps, after transmission through $96 \mathrm{~km}$ of 6-mode FMF. All 6 transmitted data streams are successfully recovered by the

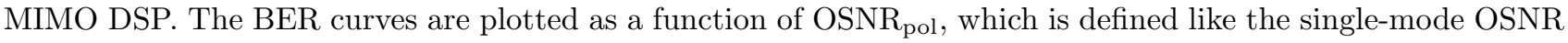
(using 0.1-nm optical noise reference bandwidth), but only the noise that is co-polarized with the corresponding 
signal component is included. Fig. 4 c) also shows the theoretical limit for coherent detection of QPSK, and the back-to-back (B2B) measurements as a reference. All B2B measurements show a penalty of less than $0.8-\mathrm{dB}$ at a BER of $10^{-3}$, and all 6 BER curves of the transmitted signals are within $1.2 \mathrm{~dB}$ from the back-toback measurements. This excellent performance shows that crosstalk present in 96-km of 6-mode FMF can be successfully compensated with very low impact on system performance.

\subsection{Impulse-response matrix measurement of the 6-mode FMF}

In Sec. 2.2 MIMO DSP techniques were applied to undo linear distortions and crosstalk introduced by the FMF. In this section, we use MIMO DSP techniques to estimate the linear transfer function of the FMF channel. The 6 -mode FMF is equivalent to a $6 \times 6$ MIMO channel, which is fully characterized by its $6 \times 6$ impulseresponses $\mathbf{h}_{n m}$, where $n$ is the index of the receive port and $m$ is the index of the transmit port. The squared magnitude of the $6 \times 6$ impulse responses are shown in Fig. 5 for the 96-km long FMF described in Sec. 2.1. In this representation, each column corresponds to the impulse responses associated to a particular transmit port, whereas each row is associated to a particular receive port. The determination of the impulse-response matrix is referred to as channel estimation in MIMO literature, and several algorithms are available. ${ }^{26}$ The results presented in Fig. 5 were obtained using a least-square-error (LSE) estimator. ${ }^{12}$ In order to highlight the components of the impulse response due to mode coupling, chromatic dispersion of $96 \times 18 \mathrm{ps} / \mathrm{nm}$ was electronically compensated on the received signal $r_{i}(k)$ prior to estimating the impulse-response matrix. This allows to clearly identify the main coupling which appears as sharp peaks. Fig. 5 is divided into four regions identified as A, B, C, and D: Region A consists of a $2 \times 2$ array located in the top left corner and is formed by the impulse-responses $h_{11}, h_{12}, h_{21}$, and $h_{21}$. Region A shows the coupling between the two polarizations of the fundamental mode $\left(\mathrm{LP}_{01}\right)$. Region $\mathrm{B}$ consists of the $4 \times 4$ array located on the bottom right corner and, comprising the impulse-responses $h_{33}, h_{36}, h_{63}$, and $h_{66}$. Region $\mathrm{B}$ represents the coupling between the spatial and polarization modes $\mathrm{LP}_{11 a}$, and $\mathrm{LP}_{11 \mathrm{~b}}$. The remaining off-diagonal regions $\mathrm{C}$ and $\mathrm{D}$, which are enclosed by $\left(h_{13}, h_{16}, h_{23}, h_{26}\right)$, and $\left(h_{31}, h_{32}, h_{61}, h_{62}\right)$, respectively, describe the crosstalk between $\mathrm{LP}_{01}$ and $\mathrm{LP}_{11}$ modes. In Fig. 5 strong coupling peaks appear in regions A and B, and typically 100 to 1000 times weaker, 2.6-ns wide distributed coupling is observed in regions $\mathrm{C}$ and $\mathrm{D}$.

The width of the distributed coupling of regions $\mathrm{C}$ and D is consistent with the DGD of 96-km 6-mode FMF, and can be interpreted as coupling occurring at various locations along the fiber. In fact, because light traveling in the faster $\mathrm{LP}_{01}$ mode will arrive earlier than light traveling in the slower $\mathrm{LP}_{11}$ mode, the time of arrival can be used to predict the location of the coupling between $\mathrm{LP}_{01}$ and $\mathrm{LP}_{11}$ modes. Note that also regions $\mathrm{A}$ and $\mathrm{B}$ show a weak distributed coupling next to the strong coupling peaks. This weaker distributed coupling represents light that couples back and forth between $\mathrm{LP}_{01}$ and $\mathrm{LP}_{11}$ or $\mathrm{LP}_{11}$ and $\mathrm{LP}_{01}$ modes, respectively.

Fig. 5 also confirms the excellent alignment of the MMUX. Any misalignment in the phase plates would be immediately visible as narrow crosstalk peaks either at the beginning or at the end of the distributed coupling in regions $\mathrm{C}$ and $\mathrm{D}$. The impulse response matrix provides a clear picture of the MIMO channel, indicating location and amount of crosstalk introduced by the MMUX and the 6-mode FMF. It is therefore a very useful tool for fiber characterization, coupler optimization, and fault localization.

\section{RAMAN AMPLIFICATION IN FEW-MODE FIBER}

Optical amplification in SDM transmission is a key enabler for successful commercial and energy efficient implementation. The strong intensity overlap between the modes of a FMF enables sharing of the amplification pump power across multiple signal modes ${ }^{28}$ similar to that observed in the amplification of multiple dense WDM channels. However, extending the benefits of optical amplification to SDM systems poses significant challenges. In fact, optical amplification in multimode fibers has been reported in Ref. 29, but a strong mode-dependent gain (MDG) was observed. Minimizing the MDG for in-line amplification is of significance because of its deleterious impact on the achievable mode multiplexed capacity. ${ }^{19}$ Bai et. al. ${ }^{30}$ proposed a scheme to control the MDG in a FMF based Erbium doped fiber amplifier (EDFA) and experimental investigations have recently followed ${ }^{13,31}$ with mixed results. In this work we present optical amplification based on the Raman effect ${ }^{32}$ in FMF, where the MDG was experimentally reduced to $<0.5 \mathrm{~dB}$. In contrast to the theoretical work on FMF-EDFAs presented in Ref. 30, our work is not based on the linearly polarized (LP) pseudo modes, ${ }^{33}$ but makes use of the true 
Transmitted ports

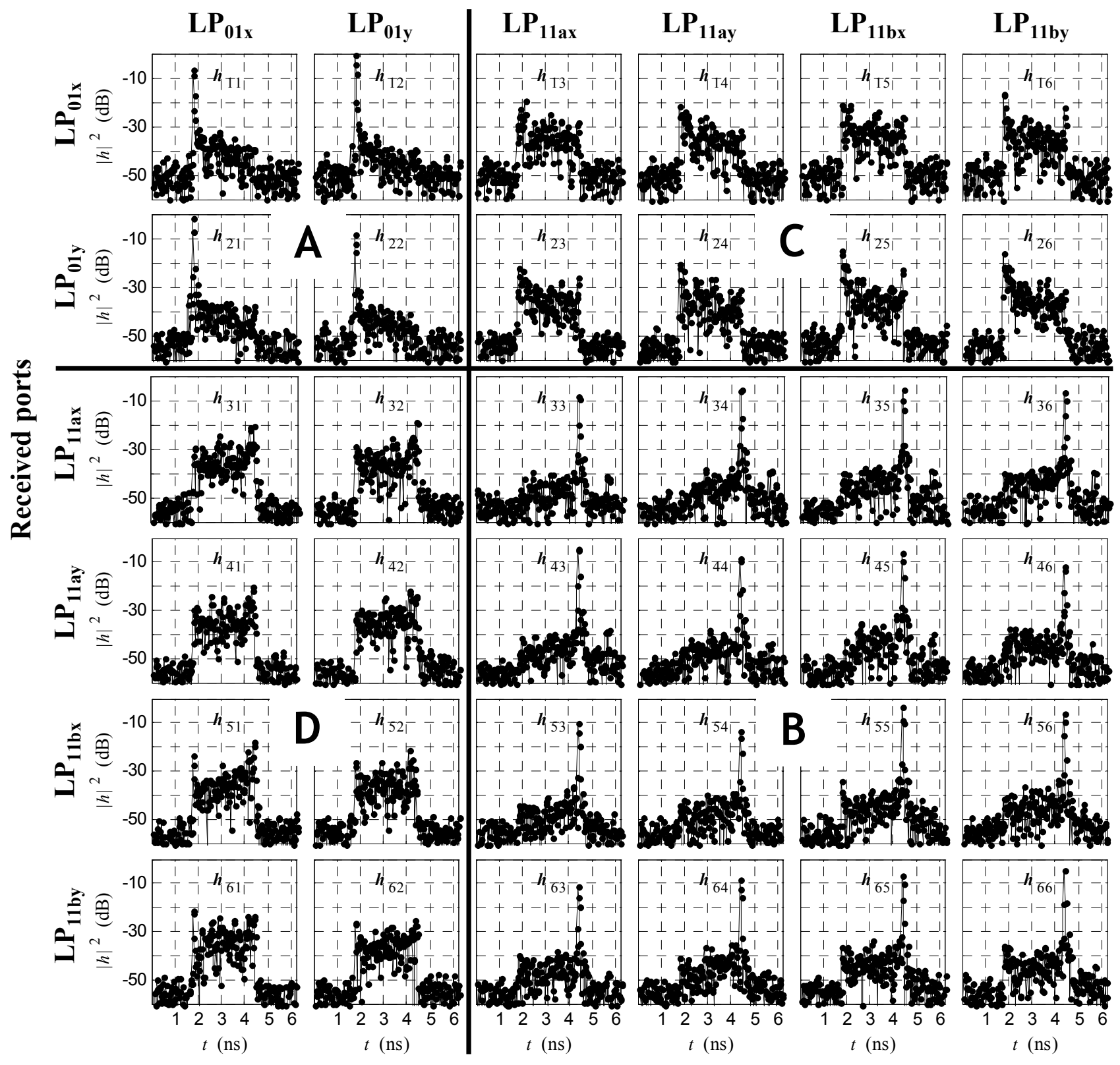

Figure 5. Squared magnitude of the PDM-SDM $6 \times 6$ impulse responses for $96 \mathrm{~km}$ of 6 -mode FMF. See text for explanation.

waveguide modes. ${ }^{34}$ For example, consider the twofold degenerate $\mathrm{LP}_{11}$ pseudomode for a SI-FMF with core diameter of $d=17 \mu \mathrm{m}$ and at a normalized frequency of $V=5$. In such a fiber the $\mathrm{LP}_{11}$ pseudomode is formed by a linear combination of the true waveguide modes $\mathrm{TE}_{01}, \mathrm{TM}_{01}$ and the two-fold degenerate $\mathrm{HE}_{21}$. The true waveguide modes forming $\mathrm{LP}_{11}$ have different values of phase velocity $\beta$, which results in a beat length of $0.31 \mathrm{~m}$ between $\mathrm{TE}_{01}$ and $\mathrm{HE}_{21}, 1.14 \mathrm{~m}$ between $\mathrm{TM}_{01}$ and $\mathrm{HE}_{21}$, and $0.24 \mathrm{~m}$ between $\mathrm{TE}_{01}$ and $\mathrm{TM}_{01}$, where $\mathrm{TE}_{01}$ has the largest and $\mathrm{TM}_{01}$ the smallest $\beta$, respectively. A launched $\mathrm{LP}_{11}$ pseudomode will therefore maintain its spatial intensity distribution along propagation only for a few $\mathrm{cm} .{ }^{35}$ Note that this effect is negligible for the transmission experiment reported in Sec. 2 because the MIMO DSP will correct for any such phase- and group-delay differences but the effect plays a critical role in optical amplification which is driven by the spatial 
intensity distribution of the pump power as well as by its spatial evolution with propagation distance.

Raman amplification transfers the power of a pump laser at $1455 \mathrm{~nm}$ to a signal around $1560 \mathrm{~nm} .{ }^{32}$ The gain is phase insensitive and polarization independent if a depolarized pump laser is used. Both pump and signal can be coupled into all modes supported by the 6 -mode FMF, and the amplification is then approximatively described by

$$
\frac{d S_{M}}{d z}=\gamma_{\mathrm{R}}\left(\sum_{N} f_{N, M} P_{N}\right) S_{M}, \text { and } \quad f_{N, M}=\frac{\iint_{-\infty}^{+\infty} I_{N}(x, y) I_{M}(x, y) d x d y}{\iint_{-\infty}^{+\infty} I_{N}(x, y) d x d y \iint_{-\infty}^{+\infty} I_{M}(x, y) d x d y}
$$

where $S_{M}$ is the power in mode $M$ at the wavelength of the signal, $P_{N}$ is the power in mode $N$ at the wavelength of the pump, $z$ is the position along the fiber, and $\gamma_{\mathrm{R}}$ is related to the cross section of spontaneous Raman scattering; $f_{N, M}$ is the intensity overlap integral and depends on the spatial intensity distributions $I_{N}$ and $I_{M}$ of the true waveguide modes of the signal signal $N$, and the pump mode $M$, respectively. Note that fast time averaging of modal interference is assumed in Eq. (3) which is valid only if the length of the amplifying fiber is much longer than the modal beat length, caused by the phase velocity differences between modes. ${ }^{36}$

A numerical analysis of Eq. (3) shows that in order to achieve equal amplification for all modes in a 6-mode FMF, the depolarized pump power has to be launched mostly into a single $\mathrm{LP}_{11}$ mode. ${ }^{15}$ This launch condition divides the pump power equally among all four true waveguide modes associated to the $\mathrm{LP}_{11}$ mode. Launched pump power into the $\mathrm{LP}_{01}$ mode is unfavorable as it produces much larger gain for the $\mathrm{LP}_{01}$ than for the $\mathrm{LP}_{11}$ signal, whereas nearly the same gain is obtained for both $\mathrm{LP}_{01}$ and $\mathrm{LP}_{11}$ modes when the pump power is launched into the $\mathrm{LP}_{11}$ mode. In our experiment the pump is counterpropagating with respect to the signal and the experimental arrangement to couple the pump and to mode multiplex the signal (Raman-MMUX) is shown in Fig. 6 a). In the Raman-MMUX, the MMUX of Fig. 2 e) is modified to separate the pump wavelength at
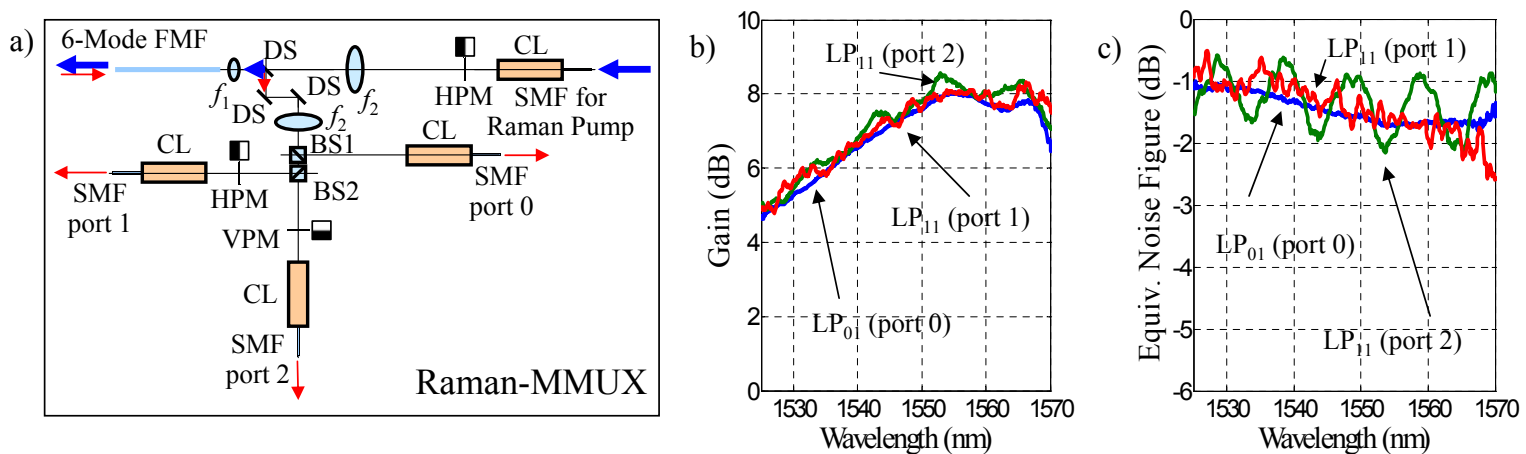

Figure 6. a) Experimental arrangement of the Raman-MMUX, showing the backward Raman pump coupler and mode demultiplexer. CL denote collimators and BS are beam splitters whereas HPM and VPN are phase plates in horizontal and vertical arrangement, respectively. b) Raman amplifier on-off gain. c) Equivalent noise figure of the Raman amplification which corresponds to the calculated noise figure a lumped optical amplifier would have when connected to the end facet of the FMF.

$1455 \mathrm{~nm}$ from the signal wavelength at $1560 \mathrm{~nm}$. This is implemented by introducing three cascaded dichroic beams splitters (DS) with a total suppression of the pump of $>72 \mathrm{~dB}$. The rest of the Raman-MMUX below the DS pair is similar to the MMUX. The Raman pump laser is coupled into the FMF after traversing a phase plates that generates an $\mathrm{LP}_{11}$ mode. This way, $1.25 \mathrm{~W}$ of pump power were coupled into the FMF starting from a 2-W pump laser, resulting in 2.04-dB loss for the pump coupler.

The Raman gain was measured by connecting 137-km 6-mode FMF between a MMUX and a Raman-MMUX. The input MMUX was connected to a broadband light source and the light received in the Raman-MMUX was detected on an optical spectrum analyzer. The measured on-off gain is plotted as a function of the wavelength in Fig. 6 b) for all three signal ports of the Raman-MMUX. The observed gain shows a maximum of $8 \mathrm{~dB}$ in 
the wavelength region between 1550 and $1570 \mathrm{~nm}$, whereas mode-dependent gain is $<0.5 \mathrm{~dB}$. The calculated equivalent noise figure for the Raman amplification in the $\mathrm{FMF},{ }^{32}$ which is equivalent to the theoretical noise figure of a corresponding lumped optical amplifier connected to the end facet of the FMF, is reported as function of the wavelength in Fig. 6 c). The equivalent noise figure is $-1.5 \mathrm{~dB}$ in the 1550 to $1570-\mathrm{nm}$ range. In this case, negative values of the noise figure are admissible and represent the fact that the amplification occurs in the FMF before the fiber loss occurs. A small but reproducible ripple in the equivalent noise figure is observed, which is also noticeable in the non amplified signal for the $\mathrm{LP}_{11}$ mode. In order to observe an 8-dB gain, a pump power of $1.25 \mathrm{~W}$ is required. The large power requirement is mostly due to the large effective area of the 6 -mode FMF. Note that there is however a real power advantage compared to using three individual SMFs. In fact, evaluating the intensity overlap integrals defined in Eq. (3) we notice that the intensity overlap integrals $f_{\left(L P_{01}\right),\left(L P_{11}\right)}$ between the fundamental mode and the true waveguide mode forming the $L P_{11}$ mode is almost the same as the intensity overlap integrals $f_{\left(L P_{11}\right),\left(L P_{11}\right)}$ between the true waveguide modes forming the $L P_{11}$ and themselves, whereas the intensity overlap integral $f_{\left(L P_{01}\right),\left(L P_{01}\right)}$ between the fundamental mode and itself is approximately 1.5 times larger than $f_{\left(L P_{11}\right),\left(L P_{11}\right)}$. This results in a power penalty for sharing the pump among all modes of a factor of 1.5, whereas the benefit compared to the use of three individual SMFs is a factor of 3. The Raman amplifier in a 6-mode FMF therefore consumes only half of the power of a SMF based system with equivalent effective area of the fundamental $\left(\mathrm{LP}_{01}\right)$ mode, this assuming an undepleted pump.

The performance of the Raman amplifier was verified by repeating the single-span transmission experiment described in Sec. 2 over a record distance of 137-km FMF. The resulting performance measured for a launch power of $5 \mathrm{dBm}$ per mode with pump on and off is very similar for received OSNR values $<16 \mathrm{~dB}$. OSNR values $>16 \mathrm{~dB}$ and $\mathrm{BER}<10^{-3}$ can only be reached with pump on, and a penalty of $6 \mathrm{~dB}$ compared to theory for a $\mathrm{BER}<10^{-3}$ is observed, confirming that the amplifier is performing as expected.

\section{SPACE-DIVISION MULTIPLEXING IN COUPLED MULTI-CORE FIBER}

Coupled-core multi-core fibers (CCFs) offer a simple way to implement SDM in an optical fiber, and if coupling between cores is allowed, cores can be placed closely, therefore increasing the density of the mode in the fiber cross section. To our knowledge the first demonstration of MIMO transmission performed over CCF with strong coupling was shown in Ref. 37, where low-penalty transmission over a distance of $24 \mathrm{~km}$ was shown for a 3-core CCF. The distance between the cores was $38 \mu \mathrm{m}$, the refractive index difference $\Delta=0.32 \%$, and a core diameter was $11.2 \mu \mathrm{m}$. More recently ${ }^{18}$ the distance between cores of the a new 3 -core CCF was reduced to $29.4 \mu \mathrm{m}$, and the core diameter was increased to $12.4 \mu \mathrm{m}$, whereas the refractive index difference was reduced to $\Delta=0.27 \%$. The resulting effective area was measured to be $(129 \pm 2) \mu \mathrm{m}^{2}$, and the total length of the fiber was $60 \mathrm{~km}$. The attenuation at $\lambda=1550 \mathrm{~nm}$ of the 3 -core $\mathrm{CCF}$ was $0.181 \mathrm{~dB} / \mathrm{km}$, and was measured by launching into a single core and receiving the output power from all cores. The cutoff wavelength of a single core was designed to be around $1350 \mathrm{~nm}$, and the chromatic dispersion and the dispersion slope were approximately $21 \mathrm{ps} / \mathrm{nm} / \mathrm{km}$ and $0.06 \mathrm{ps} / \mathrm{nm}^{2} / \mathrm{km}$, respectively, at $\lambda=1550 \mathrm{~nm}$. The crosstalk between cores was measured using a depolarized source. Compared to the fiber of Ref. 37, the distance between the cores was reduced by $25 \%$ and the effective area increased by $25 \%$. The two changes have a dramatic impact on the crosstalk between cores. In the fiber of Ref. 37 a crosstalk between cores measured using unpolarized light source, was found to be in the order of $-4 \mathrm{~dB}$. In the fiber of Ref. 18, light launched into a core is almost equally distributed among the cores after $60 \mathrm{~km}$ fiber. For such a strong crosstalk, the modes of the individual cores can no longer be considered as individual waveguides, and the mode of the structure as a whole, the "supermodes" 20,38 have to be considered. A fiber cross-section of the 3-core CCF is shown in Fig. 7 a). The calculated linearly polarized super-modes of the 3-core CCF (upper row) and their far-fields (bottom row) are shown in Fig. 7 b). The fundamental mode which is excited when all core modes have the same phase at coupling, is designated as $\mathrm{LP}_{01}$, in analogy to the features of the $\mathrm{LP}_{01}$ mode of SI-FMF. The higher-order mode, designated as $\mathrm{LP}_{11}$, is degenerate, and therefore the representation is not unique. The $\mathrm{LP}_{11 \mathrm{~b}}+i \mathrm{LP}_{11 \mathrm{~b}}$ mode shown in Fig. $7 \mathrm{~b}$ ) column 2 is obtained when a continuous phase jump of $2 / 3 \pi$ is applied to the respective core modes, whereas the second mode designated as $\mathrm{LP}_{11 \mathrm{~b}}-i \mathrm{LP}_{11 \mathrm{~b}}$ is the complex conjugated. An alternative representation for the equivalent $\mathrm{LP}_{11}$ mode is shown in columns 4 and 5 of Fig. 7 b). Note that we assume linearly polarized supermodes, and each mode has two orthogonal polarizations. Therefore, a total of 6 independent SDM transmission channels are available. 
a)

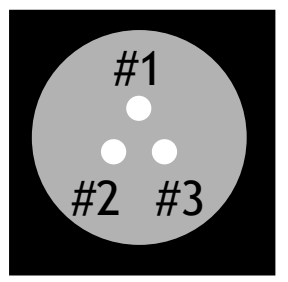

b)

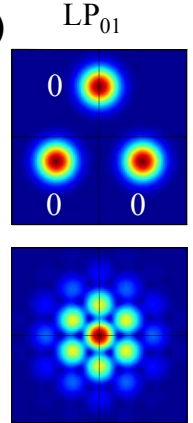

$\mathrm{LP}_{11 \mathrm{~b}}+\mathrm{LP}_{11 \mathrm{a}} \mathrm{LP}_{11 \mathrm{~b}}-\mathrm{iLP} 11 \mathrm{a}$
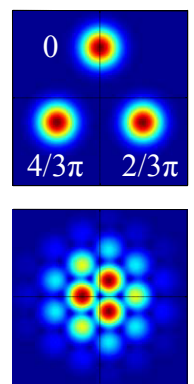
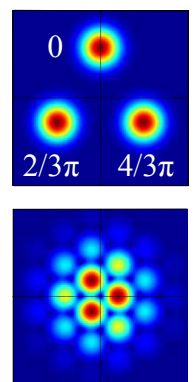

$\mathrm{LP}_{11 \mathrm{~b}}$

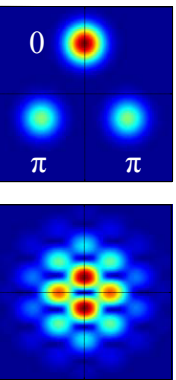

$\mathrm{LP}_{11 \mathrm{a}}$

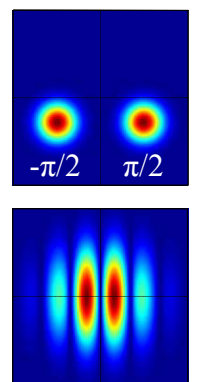

Figure 7. a) Fiber cross-section of the 3-core CCF b) Linearly polarized super-modes (upper row) and corresponding far-fields (bottom row) of the 3 -core CCF.

In order to study the MIMO transmission performance of 3-core CCFs over long distances, the setup described in Sec. 2.1 was extended to the loop experiment as shown in Fig. 8. The three PDM-QPSK input signals are

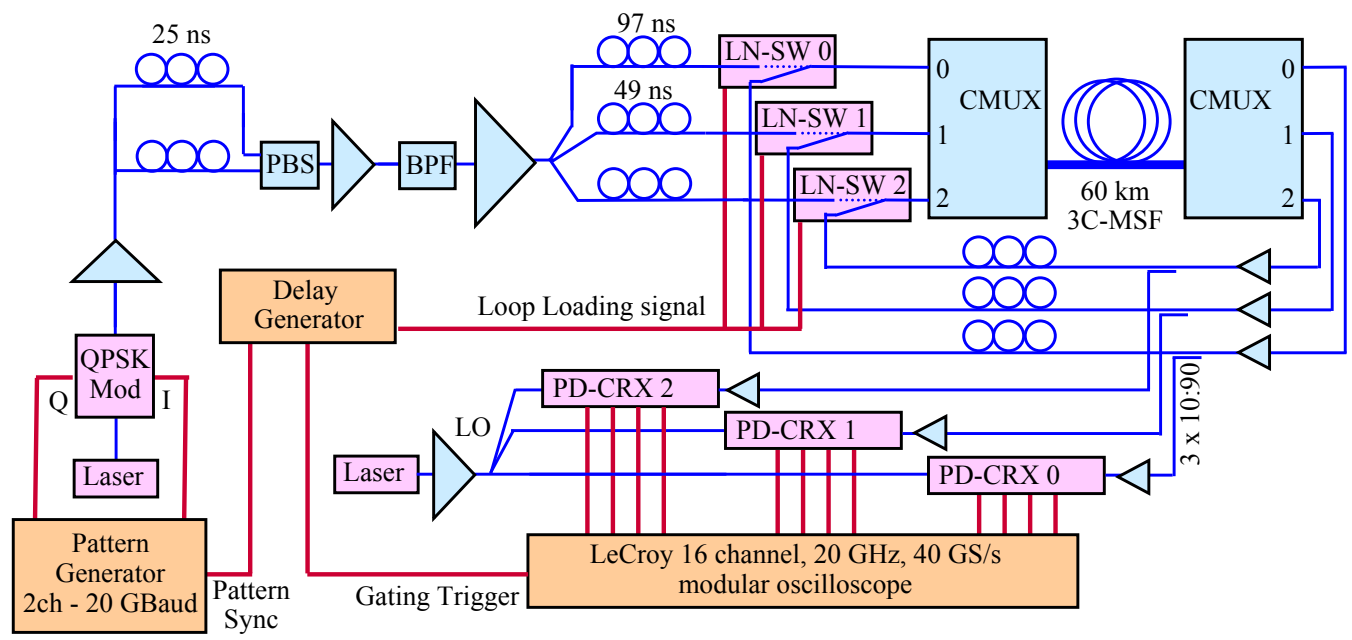

Figure 8. Experimental loop set-up. QPSK-Mod: QPSK Modulator, PBS: Polarization beam splitter, BPF: Bandpass filter, LO: Local oscillator, triangles denote EDFAs.

connected to three synchronized $\mathrm{LiNbO}_{3}$ loop switches (LN-SW), which in $2 \times 1$ configuration, inject the input signals into the parallel-loops during a 2-ms loading cycle, and subsequently close the parallel-loops for $9 \mathrm{~ms}$ when in looping configuration. The three parallel-loops consist each of a concatenation of a first core multiplexer (CMUX), followed by a 60-km 3-core CCF, a second CMUX, an EDFA, 10:90 optical couplers to extract the transmitted signal, fiber-optic delays to adjust the relative loop length, and the LN-SWs. In order to minimize the required number of taps for the MIMO DSP, the relative fiber length of the individual loops had to be matched to within $\pm 20 \mathrm{~mm}$, corresponding to a relative time delay of $\pm 100 \mathrm{ps}$. The CMUX consisted of three collimators and and imaging system and was similar to the MMUX shown in Fig. 2 e) except that the beam splitters were replaced by mirrors, since the beams are orthogonal by spatial separation. This resulted in a lower loss of $2 \mathrm{~dB}$ for all three ports.

The transmission properties for a launch power of $0 \mathrm{dBm}$ and $3 \mathrm{dBm}$ in each core were evaluated after each loop round-trip and are shown in Fig. 9 a). A BER $<10^{-3}$ was observed for up to 20 loops, corresponding to a record MIMO-transmission distance of $1200 \mathrm{~km}$. The required number of equalizer taps grows as a function of distance, as expected. After a distance of $60 \mathrm{~km}$, good performance was observed with as few as 30 taps, whereas up to 225 taps are used at maximum measured distance of $1200 \mathrm{~km}$. This experiment clearly demonstrates that both coupled multi-core fibers and MIMO digital signal processing are viable long-haul transmission technologies.

We also investigated the impulse response of the 3 -core $\mathrm{CCF}$ as a function of distance. Because all 36 impulse 

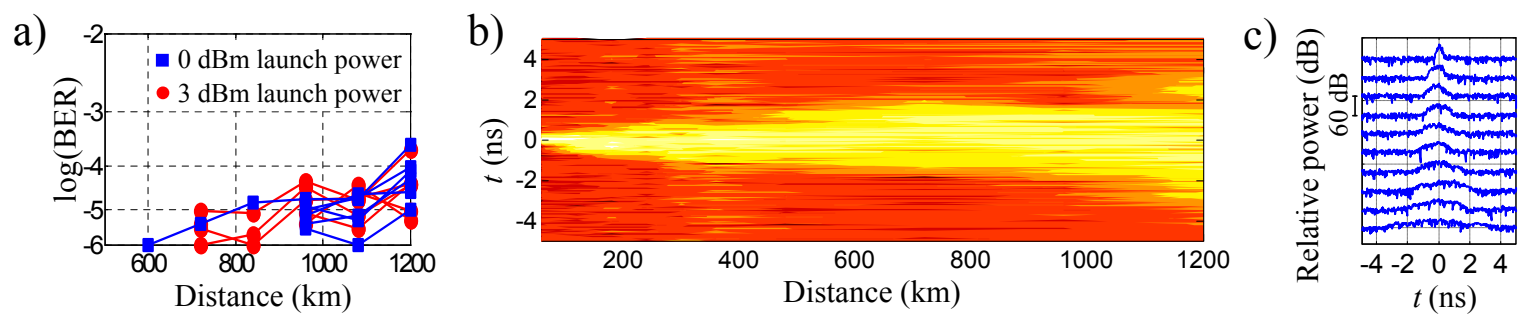

Figure 9. a) BER as function of distance for transmission over 3-core CCF. b) Squared magnitude of the impulse response in false color representation plotted as function of distance (higher magnitude is lighter colored) and c) plotted on a logarithmic scale with $60-\mathrm{dB}$ offset between the curves. The plots correspond to distances of 60, 120, 180, 240, 300, 360, 480, 720, 960, and $1200 \mathrm{~km}$ (from top to bottom).

responses of the 3-core CCF look very similar, only one impulse response is reported for every distance. The results are shown in Fig. 9 b) and c) where the squared magnitude of the impulse response after electronic dispersion compensation is shown as a function of distance using two different representations, the first using false colors (Fig. 9 b)) and the second reporting a plot of impulse response function after every loop. The impulse response has an initial pulse width of $600 \mathrm{ps}$ at a distance of $60 \mathrm{~km}$, and the pulse width clearly grows over distance reaching a width of up to $5 \mathrm{~ns}$ after $1200 \mathrm{~km}$. The observed pulse widening is sub-linear.

\section{CONCLUSION}

We demonstrated SDM transmission in both multi-mode and multi-core fibers based on MIMO digital signal processing. The results confirm that long distance transmission over both types of fiber is possible even in the presence of very large crosstalk between the SDM channels. We demonstrated single-wavelength 6-channel spatial- and polarization-mode-multiplexed transmission of $6 \times 40-\mathrm{Gb} / \mathrm{s}$ QPSK signals over $96-\mathrm{km}$ few-mode fiber with less than 1.2-dB penalty. In the same fiber and using the same modulation format we also demonstrated successful transmission over a single 137-km span based on Raman amplification. We also demonstrated the first MIMO loop experiment in multicore-fiber showing a single-wavelength 6-channel spatial- and polarizationmultiplexed transmission of $6 \times 40-\mathrm{Gb} / \mathrm{s}$ QPSK signals over $1200 \mathrm{~km}$ of 3-core multi-core fiber.

\section{ACKNOWLEDGMENTS}

We would like to thank A. Gnauck, M. Esmaeelpour, and E. Burrows for their support with the experiments, A. Sierra, S. Mumtaz, and M. Magarini for their invaluable DSP expertise, and G. Raybon, C. Xie, S. Chandrasekhar, X. Liu, and D. Kilper for support with coherent receivers and test equipment. Also we would like to thank LeCroy corporation for providing a 16-channel prototype of the LeCroy LabMaster modular digital storage oscilloscope, OFS for providing the few-mode fibers and Sumitomo Electric Industries for providing the multi-core fibers.

\section{REFERENCES}

1. Tkach, R., "Scaling optical communications for the next decade and beyond," Bell Labs Technical Journal 14(4), 3-9 (2010).

2. Essiambre, R.-J., Kramer, G., Winzer, P. J., Foschini, G. J., and Goebel, B., "Capacity limits of optical fiber networks," J. Lightwave Technol. 28, 662-701 (2010).

3. Chraplyvy, A., "The coming capacity crunch," Proc. European Conf. on Opt. Commun. (ECOC), Planary talk (2009).

4. Winzer, P. J., "Energy-efficient optical transport capacity scaling through spatial multiplexing," Photon. Technol. Lett. 23(13), 851-853 (2011).

5. Berdague, S. and Facq, P., "Mode division multiplexing in optical fibers," Appl. Opt. 21(11), 1950-1955 (1982). 
6. de Boer, M., Tsekrekos, C. P., Martinez, A., Kurniawan, H., Bergmans, J. W. M., Koonen, A. M. J., van den Boom, H. P. A., and Willems, F. M. J., "A first demonstrator for a mode group diversity multiplexing communication system," in [Proc. IEE Seminar (Ref Optical Fibre Communications and Electronic Signal Processing No. 2005-11310)], (2005).

7. Schollmann, S., Soneff, S., and Rosenkranz, W., "10.7 Gb/s over $300 \mathrm{~m}$ GI-MMF using a $2 \times 2$ MIMO system based on mode group diversity multiplexing," in [Proc. Conf. Optical Fiber Communication and the National Fiber Optic Engineers Conf. OFC/NFOEC 2007], 1-3 (2007).

8. Shah, A. R., Hsu, R. C. J., Tarighat, A., Sayed, A. H., and Jalali, B., "Coherent optical MIMO (COMIMO)," J. Lightwave Technol. 23(8), 2410-2419 (2005).

9. Li, A., Al Amin, A., Chen, X., and Shieh, W., "Reception of mode and polarization multiplexed 107Gb/s CO-OFDM signal over a two-mode fiber," Proc. Opt. Fiber Commun. Conf. (OFC), PDPB8 (2011).

10. Salsi, M., Koebele, C., Sperti, D., Tran, P., Brindel, P., Mardoyan, H., Bigo, S., Boutin, A., Verluise, F., Sillard, P., Bigot-Astruc, M., Provost, L., Cerou, F., and Charlet, G., "Transmission at 2x100Gb/s, over two modes of 40km-long prototype few-mode fiber, using LCOS based mode multiplexer and demultiplexer," Proc. Opt. Fiber Commun. Conf. (OFC), PDPB9 (2011).

11. Hanzawa, N., Saitoh, K., Sakamoto, T., Matsui, T., Tomita, S., and Koshiba, M., "Demonstration of mode-division multiplexing transmission over $10 \mathrm{~km}$ two-mode fiber with mode coupler," Proc. Opt. Fiber Commun. Conf. (OFC), OWA4 (2011).

12. Ryf, R., Randel, S., Gnauck, A. H., Bolle, C., Sierra, A. and Mumtaz, S., Esmaeelpour, M., Burrows, E. C., Essiambre, R.-J., Winzer, P. J., Peckham, D. W., McCurdy, A., and Lingle, R., "Mode-division multiplexing over $96 \mathrm{~km}$ of few-mode fiber using coherent 6 x 6 MIMO processing," J. Lightwave Technol., special issue about OFC/NFOEC 2011 .

13. Ip, E., Bai, N., Huang, Y.-K., Mateo, E., Yaman, F., Li, M.-J., Bickham, S., Ten, S., Linares, J., Montero, C., Moreno, V., Prieto, X., Tse, V., Chung, K. M., Lau, A., Tam, H.-Y., Lu, C., Luo, Y., Peng, G.-D., and Li, G., "88 x 3 x 112-gb/s WDM transmission over $50 \mathrm{~km}$ of three-mode fiber with inline few-mode fiber amplifier," Proc. European Conf. on Opt. Commun. (ECOC), Postdeadline, Th.13.C.2 (2011).

14. Koebele, C., Salsi, M., Milord, L., Ryf, R., Bolle, C., Sillard, P., Bigo, S., and Charlet, G., "40km transmission of five mode division multiplexed data streams at 100Gb/s with low MIMO-DSP complexity," Proc. European Conf. on Opt. Commun. (ECOC), Postdeadline paper, Th.13.C.3 (2011).

15. Ryf, R., Sierra, A., Essiambre, R.-J., Randel, S., Gnauck, A., Bolle, C., Esmaeelpour, M., Winzer, P., Delbue, R., Pupalaiikise, P., Sureka, A., Peckham, D. W., McCurdy, A., and Lingle Jr., R., "Mode-equalized distributed Raman amplification in 137-km few-mode fiber," Proc. European Conf. on Opt. Commun. (ECOC), Postdeadline Paper, Th.13.K.5 (2011).

16. Zhu, B., Taunay, T. F., Fishteyn, M., Liu, X., Chandrasekhar, S., Yan, M. F., Fini, J. M., Monberg, E. M., and Dimarcello, F. V., "Space-, wavelength-, polarization-division multiplexed transmission of $56 \mathrm{~Tb} / \mathrm{s}$ over a 76.8km seven-core fiber," Proc. Opt. Fiber Commun. Conf. (OFC), PDPB7 (2011).

17. Sakaguchi, J., Awaji, Y., Wada, N., Kanno, A., Kawanishi, T., Hayashi, T., Taru, T., Kobayashi, T., and Watanabe, M., "109Tb/s (7x97x172Gb/s SDM/WDM/PDM) QPSK transmission through 16.8km homogeneous multi-core fiber," Proc. Opt. Fiber Commun. Conf. (OFC), PDPB6 (2011).

18. Ryf, R., Sierra, A., Essiambre, R.-J., Gnauck, A., Randel, S., Esmaeelpour, M., S., M., Winzer, P. J., Delbue, R., Pupalaiikise, P., Sureka, A., Hayashi, T., Taru, T., and Sasaki, T., "Coherent 1200-km 6 x 6 MIMO mode-multiplexed transmission over 3-core microstructured fiber," Proc. European Conf. on Opt. Commun. (ECOC), Postdeadline Paper, Th.13.C.1 (2011).

19. Winzer, P. J. and Foschini, C. J., "MIMO capacities and outage probabilities in spatially multiplexed optical transport systems," Optics Express 19(17), 16680-16696 (2011).

20. Black, R. J. and Gagnon, L., [Optical Waveguide Modes: Polarization, Coupling and Symmetry], McGrawHill Professional (2009).

21. Thornburg, W. Q., J., C. B., and Zhu, X. D., "Selective launching of higher-order modes into an optical fiber with an optical phase shifter," Opt. Lett. 19 (7), 454-456 (1994).

22. Mohammed, W., Pitchumani, M., Mehta, A., and Johnson, E. G., "Selective excitation of the LP 11 mode in step index fiber using a phase mask," Optical Engineering 45 (7), 074602 (2006). 
23. Buck, J. A., [Fundamentals of Optical Fibers], Wiley, New York (1995).

24. Sierra, A., Randel, S., Winzer, P. J., Ryf, R., Gnauck, A. H., and Essiambre, R.-J., "On the use of delaydecorrelated I/Q test sequences for QPSK and QAM signals," Submitted to Photon. Technol. Lett. .

25. Randel, S., Ryf, R., Sierra, A., Winzer, P. J., Gnauck, A. H., Bolle, C. A., Essiambre, R.-J., Peckham, D. W., McCurdy, A., and Lingle, R., "6 x 56-Gb/s mode-division multiplexed transmission over 33-km few-mode fiber enabled by 6× 6 MIMO equalization," Optics Express 19(17), 16697-16707 (2011).

26. Benvenuto, N. and Cherubini, G., [Algorithms for communications systems and their applications], Wiley (2002).

27. Viterbi, A. J. and Viterbi, A. M., "Nonlinear estimation of PSK-modulated carrier phase with application to burst digital transmission," IEEE Trans. Inf. Theory 29, 543-551 (1983).

28. Krummrich, P. M., "Optical amplification and optical filter based signal processing for cost and energy efficient spatial multiplexing," Optics Express 19(17), 16636-16652 (2011).

29. Nykolak, G., Kramer, S. A., Simpson, J. R., DiGiovanni, D. J., Giles, C. R., and Presby, H. M., "An Erbium-doped multimode optical fiber amplifier," Photon. Technol. Lett. 3(12), 1079-1081 (1991).

30. Bai, N., Ip, E., Wang, T., and Li, G., "Multimode fiber amplifier with tunable modal gain using a reconfigurable multimode pump," Optics Exp 19(17), 16601-16611 (2011).

31. Yung, Y., Alam, S., Li, Z., Dhar, A., Giles, D., Giles, I., Sahu, J., Grüner-Nielsen, L., Poletti, F., and Richardson, D. J., "First demonstration of multimode amplifier for spatial division multiplexed transmission systems," Proc. European Conf. on Opt. Commun. (ECOC), Postdeadline Paper, Th.13.K4 (2011).

32. Bromage, J., "Raman amplification for fiber communications systems," J. Lightwave Technol. 22, 79-93 (2004).

33. Gloge, D., "Weakly guiding fibers," Appl. Opt. 10(10), 2252-2258 (1971).

34. Marcuse, D., [Theory of dielectric optical waveguides], Academic Press (1991).

35. Kogelnik, H. and Winzer, P., "Mode birefringence in weakly guided fibers," Submitted to J. Lightwave Technol. .

36. Winzer, P. J., Bromage, J., Kane, R. T., Sammer, P. A., and Headley, C., "Repetition rate requirements for time-division multiplexed Raman pumping," J. Lightwave Technol. 22(2), 401-408 (2004).

37. Ryf, R., Essiambre, R., Randel, S., Gnauck, A., Winzer, P., Hayashi, T., Taru, T., and Sasaki, T., "MIMObased crosstalk suppression in spatially multiplexed $3 \times 56-\mathrm{Gb} / \mathrm{s}$ PDM-QPSK signals for strongly-coupled 3-core fiber," Photon. Technol. Lett. 23(20) (2011).

38. Xia, C., Bai, N., Ozdur, I., Zhou, X., and Li, Guifang, L., "Supermodes for optical transmission," Optics Express 19, 16653-16664 (2011). 